\title{
Wie das Histon-Chaperon FACT aktives und stilles Chromatin bewahrt
}

MAGDALENA MURAWSKA, ANDREAS G. LADURNER

PHYSIOLOGISCHE CHEMIE, BIOMEDIZINISCHES CENTRUM (BMC), LMU MÜNCHEN

\section{Histone chaperones play a fundamental role in the regulation of} genome dynamics and stability. They are a versatile group of proteins that interact with histone proteins and govern their assembly or disassembly into nucleosomes. How the activity of histone chaperones is regulated is not well understood. Here, we discuss our new data on how histone post-transcriptional modifications cooperate with the essential histone chaperone complex FACT in active and silent regions of the genome.

DOI: $10.1007 / \mathrm{s} 12268-021-1570-\mathrm{Z}$

(c) Die Autoren 2021

Um unser zwei Meter langes Genom in einen kleinen Zellkern zu verpacken, wird die DNA um ein Oktamer positiv geladener Histonproteine gewickelt, wodurch Nukleosome entstehen. Nukleosome sind die Grund- bausteine des im Zellkern gelagerten DNAProtein-Komplexes namens Chromatin. Nukleosome sichern die ordnungsgemäße Verpackung und Integrität des Genoms, hindern aber auch die molekularen Maschinen daran, an das Erbgut zu gelangen. Folglich verwenden eukaryotische Zellen diverse Faktoren, die die Zugänglichkeit des Chromatins während aller wesentlichen und DNA-basierten Prozesse, wie der Transkription, Replikation und DNA-Reparatur, regulieren. Die Hauptmechanismen dieser Regulation umfassen:

- posttranskriptionelle Modifikationen von Histonen, die Histon-DNA-Wechselwirkungen modifizieren und von zellulären Faktoren erkannt werden,

- Histonvarianten, die die Stabilität der Nukleosome verändern können,

- ATP-abhängige Chromatin-RemodelingEnzyme, die mithilfe der ATP-Hydrolyse Nukleosome reorganisieren und

- Histon-Chaperone, die Histone binden und an dem Einbau und Austausch von Histonproteinen im Chromatin essenziell beteiligt sind (Abb. 1).

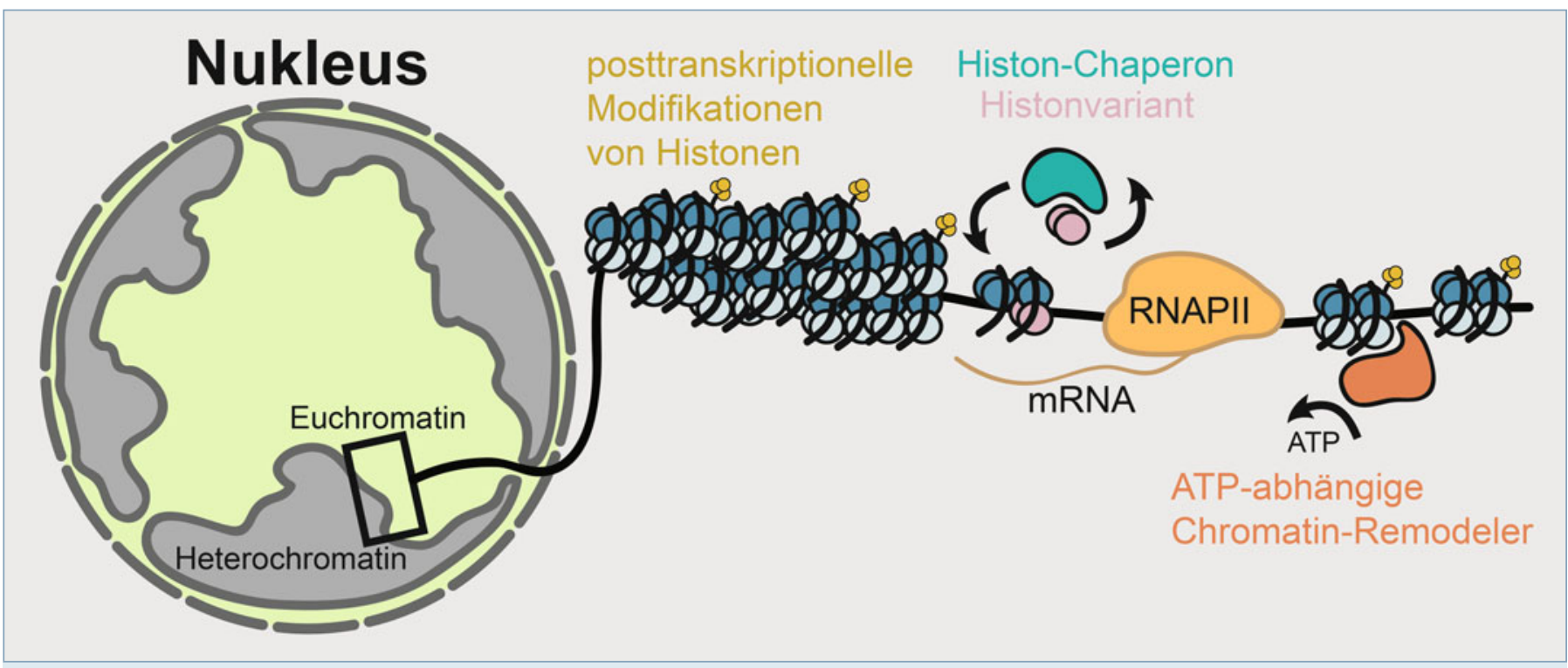

A Abb. 1: Zusammenfassung der Chromatinregulation. Ein typischer eukaryotischer Zellkern enthält transkriptionell aktives, offeneres Euchromatin (grün) und transkriptionsstilles, kompakteres Heterochromatin (grau). Nukleosome, die Hauptblöcke des Chromatins, bestehen aus Histon-Oktameren, die sich in der Regel um 147 Basenpaaren der Nukleosomen-DNA wickeln (hell- und dunkelblaue ovale Formen). Histon-Chaperone binden Histone und sind an dem Einbau und Austausch von Histonproteinen im Chromatin beteiligt. ATP-abhängige Chromatin-Remodeling-Enzyme mobilisieren oder entfernen Nukleosomen mithilfe der ATP-Hydrolyse. Histonvarianten verändern die Eigenschaften von Nukleosomen durch Änderung ihrer Stabilität. Posttranskriptionelle Modifikationen von Histonen regulieren Heterochromatin-Verdichtung (z. B. Euchromatin versus Heterochromatin). Sie rekrutieren auch verschiedene chromatinassoziierte Faktoren, die diese Modifikationen mit speziellen Proteindomänen ablesen können. Die regulatorischen Faktoren, einschließlich Histon-Chaperon, Chromatin-Remodeler, posttranskriptionelle Modifikationen der Histone, Histonvarianten und auch RNA-Polymerase II (RNAPII), sind in verschiedenen Farben dargestellt. 


\section{Histon-Chaperone tragen zur Histon- Homöostase bei}

Histon-Chaperone sind vielfältige Proteine, die die Assemblierung und Disassemblierung von Nukleosomen kontrollieren, indem sie zufällige und schädliche Wechselwirkungen zwischen stark negativ geladenen Histonen mit anderen zellulären Komponenten verhindern. Histon-Chaperone stellen auch die Aufrechterhaltung epigenetischer Zustände durch den korrekten räumlichen Einbau von Histonvarianten und modifizierten Histonen sicher. Folglich sind sie essenziell für die Aufrechterhaltung der Genomstabilität und der zellulären Identität. In der Tat liegt bei diversen Krebsarten eine Fehloder Überexpression von bestimmten Histon-
Chaperonen vor. Auch tritt während des Alterns und der zellulären Seneszenz ein Histonabbau auf [1]. Histon-Chaperone spielen daher eine Schlüsselrolle bei der Aufrechterhaltung der Histon-Homöostase, die für die Zellgesundheit wesentlich ist.

Typischerweise ist die Histon-ChaperonAktivität unabhängig von ATP-HydrolyseReaktionen, sodass Histon-Chaperone nicht aktiv im Sinne einer Maschine die Chromatinstruktur aufrechterhalten. Vielmehr binden Histon-Chaperone ihre Histonsubstrate über stark negativ geladene Domänen oder unstrukturierte Proteinregionen. Einige Chaperone sind bestimmten Histonvarianten zugeordnet, während andere Histon H3-H4-Tetramere oder H2A-H2B-Dimere

\section{A}

\section{Genkörper}

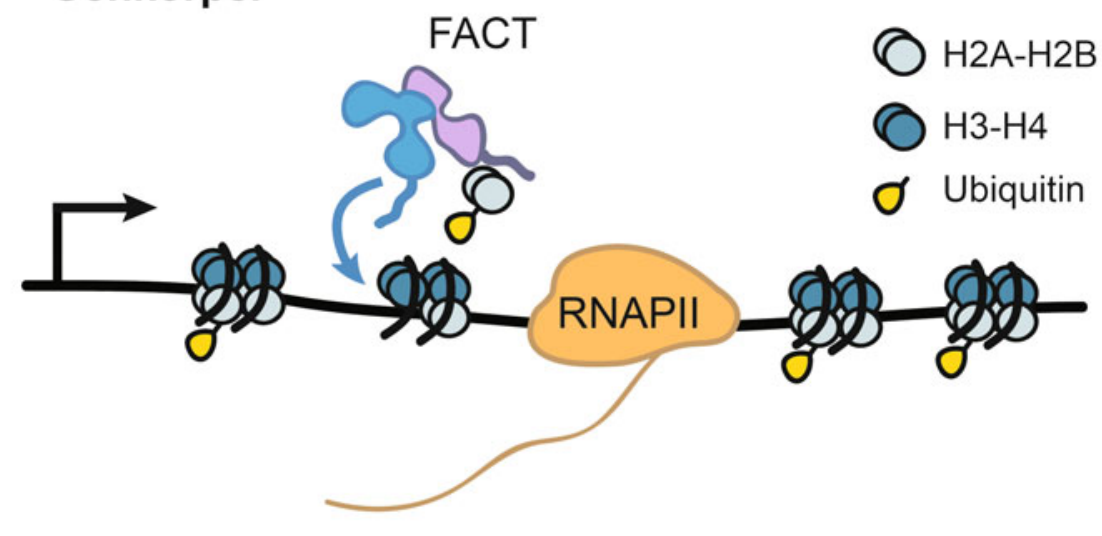

B

\section{Subtelomerer Knopf}

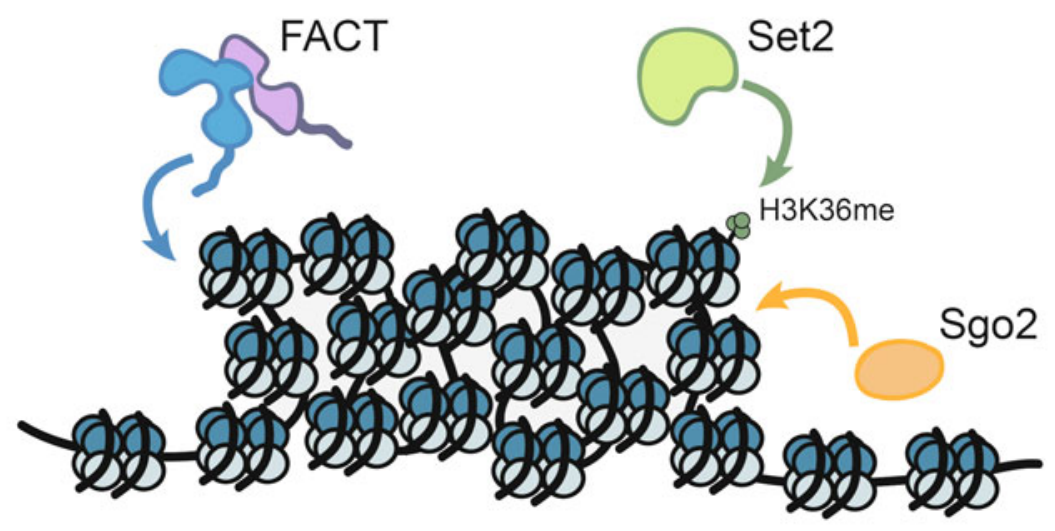

$\Delta$ Abb. 2: Zusammenfassung der FACT-Chaperonfunktionen in Genkörpern und subtelomeren Knöpfen. A, In den Genkörpern aktiver Gene kooperieren FACT und H2Bub beim Zusammenbau von Nukleosomen nach der Transkription. RNAPII: RNA-Polymerase II. B, In den verdichteten subtelomerischen Knöpfen kooperiert FACT mit der H3K36-Methylierung beim Aufbau von hochverdichtetem Chromatin. Set2: H3K36-Methyltransferase; Sgo2: centromerisches und subtelomerisches Protein. binden können. Viele Histon-Chaperone arbeiten in verschiedenen Schritten der Transkription oder Replikation zusammen, jedoch ist es noch nicht bekannt, wie genau ihre Aktivität reguliert und koordiniert wird.

\section{Der essenzielle FACT-Komplex}

Unser Labor ist an einem der evolutionär konserviertesten und auch essenziellen Histon-Chaperone interessiert, dem FACT(facilitates chromatin transcription/ transactions)-Komplex. FACT ist ein Proteinkomplex, der aus zwei Untereinheiten, Spt16 und Pob3/Ssrp1, besteht. Der humane FACTKomplex wurde als ein biochemischer Faktor identifiziert, der in vitro für die Transkription des Chromatins durch die RNA-Polymerase II benötigt wird [2]. FACT ist ein vielseitiges Histon-Chaperon mit verschiedenen Aktivitäten. FACT kann sowohl H3-H4-, als auch H2A-H2B-Histone über verschiedene Domänen des dimeren Komplexes binden. Eine neue Kryo-EM-Struktur von FACT im Komplex mit einem Teil eines Nukleosoms bestätigt frühere in vitro-Studien, die zeigten, dass FACT mit H2A-H2B-Dimeren um die nukleosomale DNA-Bindung konkurriert [3, 4]. Dieser wichtige, dynamische Schritt ist bei der reversiblen Assemblierung und Disassemblierung von Nukleosomen durch FACT von grundlegender Bedeutung. Wie FACT diese verschiedenen Aufgaben ausführt und ob seine Assemblierung- oder Disassemblierungaktivitäten und die Nutzung bestimmter Histonsubstrate vom zellulären Umfeld abhängig sind, ist derzeit noch unbekannt. Allerdings deuten erste Versuche mit FACTspezifischen niedermolekularen Inhibitoren darauf hin, dass der FACT-Komplex besonders in aktiv prolifierenden Krebszellen eine bedeutendere Rolle spielt.

\section{FACT und Histon-2B-Monoubiquiti- nierung kooperieren}

Unsere neuesten Studien beleuchten die Rolle von Histonmodifikationen bei der Regulation von FACT in der Spalthefe Schizosaccharomyces pombe, einem sehr hilfreichen Modellorganismus. Die Bindung von FACT an Gene in der Spalthefe korreliert, ähnlich wie bei anderen Organismen, mit Regionen aktiver Transkription. FACT reichert sich dementsprechend vor allem im Inneren transkribierter Gene an. Neue Studien legen nahe, dass das partielle Demontieren der Nukleosome während der Transkription durch die RNA-Polymerase II (RNAPII) His- 
tonsubstrate erzeugt, die von FACT erkannt werden und zur Rekrutierung des Chaperons an den Genkörper führt [5]. Die H2BMonoubiquitinierung (H2Bub) ist eine kovalente Modifikation von H2B, die sich ebenfalls im Geninneren ansammelt und an der Transkriptionsverlängerung beteiligt ist. Interessanterweise zeigten frühe Studien, dass sowohl H2Bub als auch FACT die RNAPII-Transkription durch Chromatin in vitro stimulieren [2].

Wie FACT und H2Bub möglicherweise in vivo zusammenarbeiten, wurde jedoch nicht verstanden. Wir untersuchten daher die Nukleosomenverteilung in den H2Bubund FACT-Mutanten mit einem genomweiten MNase-seq-Ansatz. Diese Methode beruht auf der Verwendung der Mikrokokken-Nuklease (MNase), die Linker-DNA zwischen den Nukleosomen schneidet. Danach wird die geschützte nukleosomale DNA isoliert und sequenziert. Wir fanden heraus, dass sich in einem Hefestamm ohne H2Bub weniger Nukleosome im Genkörper befinden, was dafür spricht, dass H2Bub bei der Wiederherstellung der Nukleosome nach der RNAPIIPassage beteiligt ist.

Zusätzlich zeigte eine RNA-seq-Analyse eine erhöhte Antisense-Transkription in den Genen der H2Bub-Mutante. Unerwarteterweise fanden wir, dass die Depletion von FACT in der H2Bub-Nullmutante sowohl die Nukleosome als auch die Antisense-Transkription im Geninneren wiederherstellt. Zusätzlich zeigten Ko-Immunpräzipitationsexperimente, dass in der H2Bub-Nullmutante mehr H2A-H2B-Histone mit FACT assoziiert sind [6]. Diese Ergebnisse legen nahe, dass die FACT-Bindung an Histone in der Abwesenheit von H2Bub verändert ist. In vitroExperimente mit dem rekonstituierten FACTKomplex und H2Bub-modifizierten Histonen zeigten außerdem, dass die H2B-Ubiquitinierung die Aktivität der FACT-Nukleosomenassemblierung stimuliert.

Zusammengenommen legen unsere in vivo- und in vitro-Ergebnisse nahe, dass H2Bub bei der Assemblierung von Nukleoso- men in den Genkörpern hilft, indem es die Freisetzung von H2A-H2B-Dimeren vom FACT-Komplex erleichtert (Abb. 2A, [6]). Wir denken, dass H2Bub die Histon-ChaperonDynamik erhöht, was wiederum wichtig ist, um die Histonablagerung auf der DNA und den anschließenden Zusammenbau der Nukleosome zu erleichtern.

Wie aber kann die H2B-Ubiquitylierung die Histon-Chaperon-Bindung reduzieren? H2Bub als sterisch anspruchsvolle posttranskriptionelle Modifikation könnte z. B. den Zugang zu Epitopen verdecken, die für die Histon-Chaperon-Bindung wichtig sind. Eine andere Möglichkeit wäre, dass das ubiquitinierte Chromatin eine physikalische Umgebung schafft, die die Dynamik von HistonChaperon-Wechselwirkungen verbessert. In Übereinstimmung mit dieser Hypothese stört H2Bub die Chromatinverdichtung in vitro und führt zu offeneren und zugänglicheren Chromatinfasern [7]. Eine Flüssigphasentrennung könnte ebenfalls an diesem Prozess beteiligt sein [8].

\section{Hier steht eine Anzeige.}

\section{Springer}




\section{FACT und H3K36me erleichtern Chromatinstrukturen höherer Ordnung}

Ein weiteres unerwartetes Ergebnis unserer Studien impliziert die Rolle von FACT bei der Bildung von Chromatinstrukturen höherer Ordnung. In S. pombe sind die subtelomerischen Chromosomenregionen in hochverdichteten Strukturen organisiert, die als „Knöpfe“ bezeichnet werden (Abb. 2B, [9]). Die physiologische Funktion dieser Knöpfe am Chromosomenende ist nicht gut verstanden, jedoch befinden sich viele meiotische Gene in diesen Genomregionen. Meiotische Gene müssen während des gesamten mitotischen Zellzyklus ausgeschaltet sein und dürfen erst beim Eintritt in die Meiose gezielt aktiviert werden. Während der Meiose verschwinden nämlich die Knöpfe, was indirekt ihre Beteiligung an der Stummschaltung der meiotischen Gene impliziert [9]. In den FACT-Mutanten sind die Knöpfe um etwa 50 Prozent reduziert, subtelomere Gene werden entsprechend aktiviert und die Anzahl an Nukleosomen in den Knöpfen ist deutlich verringert [6]. Somit trägt FACT zur Bildung hochverdichteter subtelomerer Regionen bei.

Unsere genetische Analyse in S. pombe ergab, dass FACT bei der Knopfassemblierung mit der Histon-3K36-Methylierung zusammenarbeitet. Es wurde zuvor gezeigt, dass diese posttranskriptionelle Modifikation zusammen mit einem centromerischen und subtelomerischen Protein, Shugoshin (Sgo2), für die Knopfbildung wichtig ist (Abb. 2B, [10]). Der genaue molekulare Mechanismus der Knopfbildung ist jedoch unbekannt. Durch die Aufrechterhaltung der Nukleosomenstruktur in Subtelomeren könnte FACT dazu beitragen, andere Faktoren zu rekrutieren, wie beispielsweise Sgo2 [10]. Es ist auch möglich, dass FACT eine direktere Rolle bei der Chromatinbildung höherer Ordnung spielt. In einem rekonstruierten System wurde zuvor gezeigt, dass FACT die mitotische Chromosomenverdichtung erleichtert [11]. Darüber hinaus hat eine kürzlich durchgeführte Studie herausgefunden, dass FACT mit Cohesin assoziiert und an der dreidimensionalen räumlichen Organisation des Genoms beteiligt ist [12]. Es wäre daher hochinteressant zu untersuchen, wie FACT an der räumlichen Chromatinorganisation und der Strukturbildung höherer Ordnung beteiligt ist.

\section{Perspektiven}

Obwohl unser Wissen über den FACT-Komplex im letzten Jahrzehnt stark erweitert wurde, bleiben viele Fragen offen. Sehr wenig ist z. B. über die Rolle dieses hochkonservierten Chaperons bei der Regulation von Chromatinzuständen während der Evolution von Metazoen bekannt. Warum ist FACT in fast allen Arten essenziell und in einigen wenigen wieder nicht? Wir verstehen derzeit auch nicht, warum Krebszellen den FACTKomplex überproduzieren und wie ein einzelner Chaperonkomplex so viele verschiedene Prozesse auf Chromatinbasis ausführen und koordinieren kann. Mit der CRISPRTechnologie, ausgefeilten Genom- und Einzelzellansätzen sowie verbesserten Kryo-EMMethoden freuen wir uns auf viele weitere, auch unerwartete Entdeckungen dieses faszinierenden Komplexes.

\section{Danksagungen}

Wir danken Charlotte Blessing für die Korrektur des Texts. Diese Forschung wurde von der Europäischen Kommission (Marie-Curie Individual Fellowship H2020-MSCA-IF-2014, 657244 für M.M.), Friedrich-Baur-Stiftung (M.M.) sowie DFG SFB1064 (Projekt-ID 213249687) und DFG SFB1309 (Projekt-ID 325871075) (A.G.L.) unterstützt.

\section{Literatur}

[1] Nye J, Melters DP, Dalal Y (2018) The art of war: harnessing the epigenome against cancer. F1000Res 7: 141

[2] Orphanides G, LeRoy G, Chang CH et al. (1998) FACT,

a factor that facilitates transcript elongation through nucleosomes. Cell 92: 105-116

[3] Liu Y, Zhou K, Zhang N et al. (2020) FACT caught in the act of manipulating the nucleosome. Nature 577: 426-431
[4] Kemble DJ, McCullough LL, Whitby FG et al. (2015) FACT disrupts nucleosome structure by binding H2A-H2B with conserved peptide motifs. Mol Cell 60: 294-306

[5] Formosa T, Winston F (2020) The role of FACT in managing chromatin: disruption, assembly, or repair? Nucleic Acids Res 48: 11929-11941

[6] Murawska M, Schauer T, Matsuda A et al. (2020) The chaperone FACT and histone H2B ubiquitination maintain S. pombe genome architecture through genic and subtelomeric functions. Mol Cell 77: 501-513

[7] Fierz B, Chatterjee C, McGinty RK et al. (2011) Histone H2B ubiquitylation disrupts local and higher-order chromatin compaction. Nat Chem Biol 7: 113-119

[8] Gallego LD, Schneider M, Mittal C et al. (2020) Phase separation directs ubiquitination of gene-body nucleosomes. Nature 579: 592-597

[9] Matsuda A, Chikashige Y, Ding D-Q et al. (2015) Highly condensed chromatins are formed adjacent to subtelomeric and decondensed silent chromatin in fission yeast. Nat Commun 6: 7753

[10] Tashiro S, Handa T, Matsuda A et al. (2016) Shugoshin forms a specialized chromatin domain at subtelomeres that regulates transcription and replication timing. Nat Commun 7: 10393

[11] Shintomi K, Takahashi TS, Hirano T (2015)

Reconstitution of mitotic chromatids with a minimum set of purified factors. Nat Cell Biol 17: 1014-1023

[12] Garcia-Luis J, Lazar-Stefanita L, Gutierrez-Escribano P et al. (2019) FACT mediates cohesin function on chromatin. Nat Struct Mol Biol 26: 970-979

Funding note: Open Access funding enabled and organized by Projekt DEAL. Open Access: Dieser Artikel wird unter der Creative Commons Namensnennu 4.0 International Lizenz veröffentlicht, welche die Nutzung, Vervielfältigung, Bearbeitung, Verbreitung und Wiedergabe in jeglichem Medium und Form erlaubt, sofern Sie den/die ursprunglichen Autor(en) und die Quelle
ordnungsgemäß nennen, einen Link zur Creative Commons Lizenz beifügen und
angeben, ob Änderungen vorgenommen wurden. Die in diesem Artikel angeben, ob Änderungen vorgenommen wurden. Die in diesem Artikel genannten Creative Commons Lizenz, sofern sich aus der Abbildungslegende nichts anderes ergibt. Sofern das betreffende Material nicht unter der genannten Creative Commons Lizenz steht und die betreffende Handlung nicht nach gesetzlichen Vorschriften erlaubt ist, ist für die oben aufgeführten Weiterverwendungen des Materials die Einwilligung des jeweiligen Weiterverwendungen des Materials die Einwilligung des jeweiligen
Rechteinhabers inzuholen. Weitere Details zur Lizenz entnehmen Sie bitte der
Lizenzinformation auf http://creativecommons.org/licenses/by/4.0/deed.de.

Korrespondenzadresse:

Dr. Magdalena Murawska

Physiologische Chemie

Biomedizinisches Centrum München

Ludwig-Maximilians-Universität

Großhaderner Straße 9

D-82152 Planegg-Martinsried

magdalena.murawska@bmc.med.Imu.de

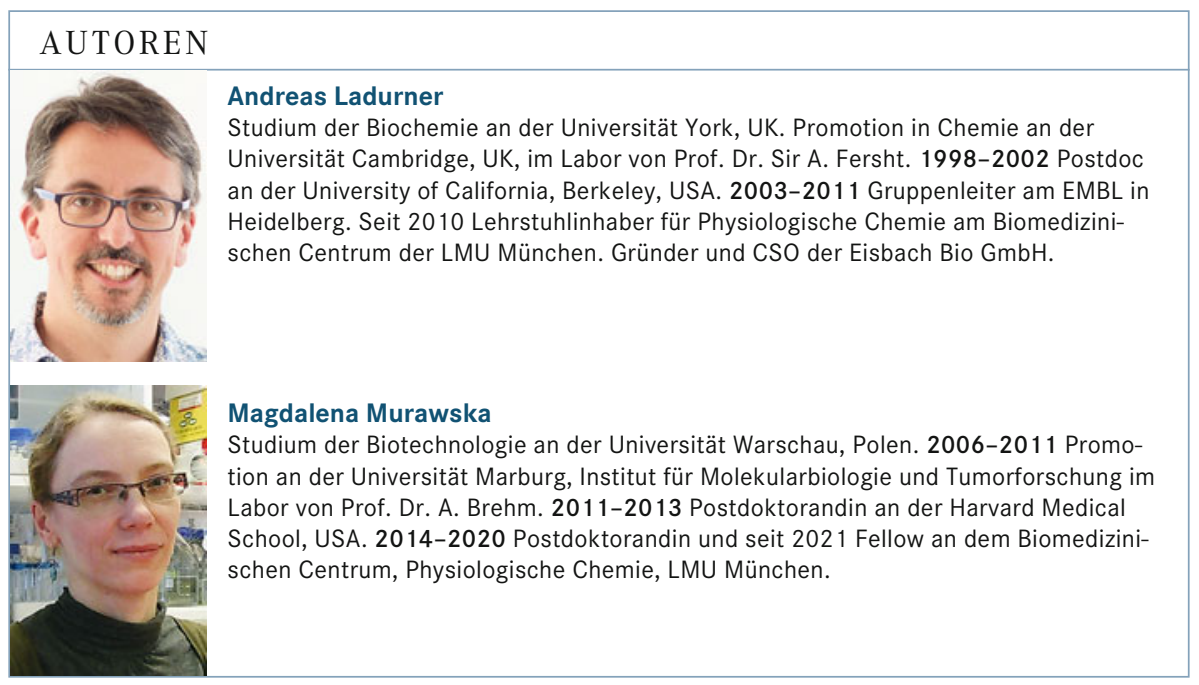

